\title{
SPECT/CT in neuroendocrine tumours
}

\author{
Orazio Schillaci • Angela Spanu • Barbara Palumbo • \\ Roberta Danieli
}

Received: 8 September 2014/Accepted: 20 November 2014/Published online: 6 December 2014

(C) Italian Association of Nuclear Medicine and Molecular Imaging 2014

\begin{abstract}
Neuroendocrine tumours (NETs) are a heterogeneous group of neoplasms with very varying clinical expressions. Nuclear medicine plays an important role in the imaging and treatment of these tumours. Despite the advent of several PET radiopharmaceuticals, SPECT imaging with the labelled somatostatin receptor analogue In-111 pentetreotide or with radioiodinated MIBG is still a very useful technique for evaluating patients with NETs. The aim of this review is to summarise the applications, in the study of NETs, of hybrid SPECT/CT imaging performed using these gamma-emitting radiopharmaceuticals. The combined use of SPECT and CT in hybrid devices is of value when applied to scintigraphic procedures in NETs: the addition of anatomical maps provides a precise localisation of SPECT findings and allows the exclusion of disease in sites of physiological tracer uptake. These advantages translate into better sensitivity and specificity, both of which are essential for the diagnosis and accurate
\end{abstract}

Color figures online at http://link.springer.com/article/10.1007/ s40336-014-0091-x.

O. Schillaci $(\varangle) \cdot$ R. Danieli

Department of Biomedicine and Prevention, University Tor

Vergata, Rome, Italy

e-mail: orazio.schillaci@uniroma2.it

O. Schillaci

IRCCS Neuromed, Pozzilli, Italy

A. Spanu

Section of Nuclear Medicine, Department of Clinical and

Experimental Medicine, University of Sassari, Sassari, Italy

B. Palumbo

Section of Nuclear Medicine and Health Physics, Department of Surgical and Biomedical Sciences, University of Perugia, Perugia, Italy staging of NETs. SPECT/CT hybrid images are able to provide additional information that improves the diagnostic accuracy and confidence of SPECT interpretation and leads to changes in therapeutic options in about $25 \%$ of patients. Therefore, SPECT/CT should be routinely performed and considered standard practice in the diagnostic work-up and treatment planning of patients with NETs submitted to In111 pentetreotide or radioiodinated MIBG imaging. This approach can still be used as an alternative to PET/CT options when the latter are not available.

Keywords Neuroendocrine tumours · Hybrid imaging · SPECT/CT $\cdot$ In-111 pentetreotide $\cdot$ MIBG

\section{Introduction}

Neuroendocrine tumours (NETs) are rare neoplasms that are derived from neuroendocrine cells interspersed throughout the body [1]. About $70 \%$ of these tumours derive from the gastroenteropancreatic system and their incidence appears to be rising; in fact, an analysis of the Surveillance, Epidemiology and End Results database indicates an increase in the reported annual age-adjusted incidence of NETs from $1.09 / 100,000$ in 1973 to $5.25 /$ 100,000 in 2004 [2]. This may be in part due to improvements in imaging and biochemical methods of detection.

NETs are well known for producing various hormonal syndromes and for their indolent clinical course in most patients, although some of these tumours do not produce hormones of clinical significance [3]. Patients may have symptoms for many years before the diagnosis is suspected and confirmed; symptoms can be caused by hormonal excess, local tumour growth, or metastatic spread [4]. A well-known characteristic of NET cells is the expression of 
several receptors in high quantities [5]. Apart from location, NETs are also graded according to proliferation activity, often evaluated by the Ki-67 labelling index, which can a have strong impact on prognosis and therapy [6].

Conventional imaging of NETs is often difficult, because of the small lesion size, variable anatomical location, and low metabolic rate; computed tomography (CT), ultrasound, and magnetic resonance imaging (MRI) are often unable to characterise and sometimes unable to visualise them [5]. Therefore, functional imaging plays an important role in evaluating patients with NETs: nuclear medicine radiopharmaceuticals emitting single photons and positrons allow accurate molecular imaging of NETs, complementary to anatomical-morphological techniques such as CT and MRI [7, 8]. In particular, expression of somatostatin receptors by NETs has been exploited for both diagnostic and therapeutic purposes $[8,9]$. Because most NETs overexpress somatostatin receptors, they can be successfully visualised in vivo by somatostatin receptor imaging, which has improved their detection [10]. Scintigraphic exams with radiolabelled somatostatin analogues (somatostatin receptor scintigraphy, SRS) and metaiodobenzylguanidine (MIBG) give important information on the receptor expression, functional status, metabolism, and tissue viability of these neoplasms [10,11]. Therefore, NET imaging clearly requires a multimodality approach with combined use of both anatomical and functional examinations. This is best achieved using hybrid imaging devices $[12,13]$.

Several clinical studies have clearly demonstrated that In-111 pentetreotide, which is the most widely used somatostatin receptor SPECT radiopharmaceutical, is effective in the diagnosis and staging of somatostatin receptor-positive tumours, especially gastroenteropancreatic (GEP) neuroendocrine ones [14-18]. The acquisition of SPECT imaging, when compared with planar scans, has proved very useful, especially in the case of tumours that are small, located in the abdomen and not visualised on planar scans, as an over-projection by other tissues and/or organs (liver, spleen, kidneys, and intestines) which show some variable individual accumulation of the radiopharmaceutical [19, 20]. However, in the interpretation of SPECT studies, it can be difficult to localise the precise anatomical sites of accumulation of the radiotracers. Moreover, although SPECT is able to improve the possibility of localising a focus of abnormal accumulation in cross-sections, normal activity-containing structures may be more difficult to identify. By combining the anatomical localising capability of CT with SPECT data, through image fusion with hybrid imaging systems, disease sites can be identified more accurately [21].

MIBG radiolabelled with I-123 or I-131 plays its major role in patients with pheochromocytomas, neuroblastomas, and paragangliomas [11]. In 1997, Perault et al. [22] described a simple non-invasive retrospective technique for registering and superimposing $\mathrm{CT}$ and SPECT images in the thoracoabdominal region in patients with endocrine tumours, without the use of external markers. This series included three cases of carcinoid tumours evaluated with In-111 pentetreotide or I-131 MIBG. Their preliminary findings suggested that SPECT/CT image fusion might be of value in improving the accuracy of SPECT alone in detecting and localising tumour recurrence or metastasis, thus affecting the management of patients with endocrine carcinomas. Subsequently, the use of fusion of I-131 MIBG SPECT images with MRI to accurately identify metastatic lesions in a patient with a malignant pheochromocytoma was reported [23]. Hybrid SPECT/CT may be of value in providing a better localisation of tumour sites, especially in the vicinity of normal organs with high MIBG uptake (i.e. liver and myocardium), and in characterising areas of normal MIBG biodistribution or excretion, thus alleviating the need for delayed images [24]. Moreover, SPECT/CT may also improve the quantification of radiation burden in patients receiving I-131 MIBG therapy [25].

The aim of this review, based on a comprehensive PubMed/MEDLINE search for studies from 2001 up to June 2014, is to illustrate, with reference to patients with NETs, the development of hybrid SPECT/CT using In-111 pentetreotide and/or MIBG, and its applications in diagnostic imaging.

\section{In-111 pentetreotide SPECT/CT}

The clinical value of the new technology of combined emission and transmission tomography using a SPECT/CT hybrid device was first assessed by Even-Sapir et al. [26] in 13 patients with NETs, using In-111 pentetreotide $(n=10)$ or I-123 MIBG $(n=3)$. Hybrid imaging findings were compared with the results of surgery and with clinical and radiological follow-up. Fused images were useful for SPECT interpretation in six patients (i.e. accurate localisation of lesions, detection of bone involvement, localisation of physiological uptake), and provided information of clinical value in four, assisting with better planning of surgery in two patients and changing the treatment approach in the other two.

The same group then retrospectively studied 72 patients with NETs to evaluate the impact of sequentially performed SPECT/CT fusion on SRS study interpretation and clinical management of these tumours [27]. Additional data for image interpretation provided by fused images as compared to SPECT alone were recorded. SPECT/CT data were verified with surgical findings when available, and with clinical and radiological follow-up. SRS studies were negative in 28 patients and positive in 44 cases; SPECT/CT 
provided no additional information in 49 patients, including all 28 negative studies. In $52 \%$ of the patients with abnormal scintigraphic findings, SPECT/CT improved the accuracy of the SRS studies by providing better localisation of SPECT-detected lesions; in particular, in 17 patients it precisely defined the organ involved and the relationship of lesions to adjacent structures, in three cases it showed unsuspected bone involvement, and in three patients it differentiated physiological from tumour uptake. It is worth noting that these outcomes affected the clinical management in 10 patients: they altered the surgical approach in six patients, spared two patients unnecessary surgery, and modified the therapeutic modality in two patients.

Fifty-four patients with known or suspected NETs were prospectively studied by Pfannenberg et al. [28] with In111 pentetreotide $(n=43)$ or I-123 MIBG $(n=13)$ using SPECT/CT and contrast-enhanced CT. The standard of reference was either histopathology or clinical and imaging follow-up data. In 56 of the 114 evaluated lesions (49\%), the results of SPECT and CT were concordant: all lesions were interpreted as malignant. However, in 58 of the 114 lesions (51\%), consensus reading of fused images changed the initial image classification. Thirty-one lesions, originally interpreted as equivocal $(n=10)$ or benign $(n=21)$, were re-classified as malignant, and 27 lesions originally interpreted as equivocal $(n=19)$ or malignant $(n=8)$ were re-classified as benign. Improved lesion classification was due mainly to correct identification of small hepatic lesions or normal-sized lymph nodes on CT, or detection of tumour foci in the event of missing or increased physiological radiopharmaceutical uptake on scintigraphy. This higher accuracy of SPECT/CT in classifying NET lesions and the results of image fusion caused a change in treatment approach in a substantial proportion $(28 \%)$ of patients. In fact, in five patients tumour could be excluded, and in four the surgical approach was changed owing to precise tumour localisation and minimisation of the surgical field; three patients were spared unnecessary surgery because of additional lesions indicating systemic disease spread, and in two patients, medical and peptide receptor radionuclide therapy was changed.

Our preliminary findings in 19 patients with GEP tumours had indicated that SPECT/CT was able to improve image interpretation and accuracy in a significant percentage of cases (47\%), thus enhancing the role of In-111 pentetreotide SPECT in this clinical setting [24]. The standard of reference was either histopathology or clinical and imaging follow-up data. In Queryparticular, in our prospective study, anatomical-functional images revealed unsuspected bone metastases in two patients (Fig. 1), providing a correct localisation of SPECT findings in six cases (Fig. 2) and disease exclusion in sites of physiological radiopharmaceutical uptake in one patient (Fig. 3).
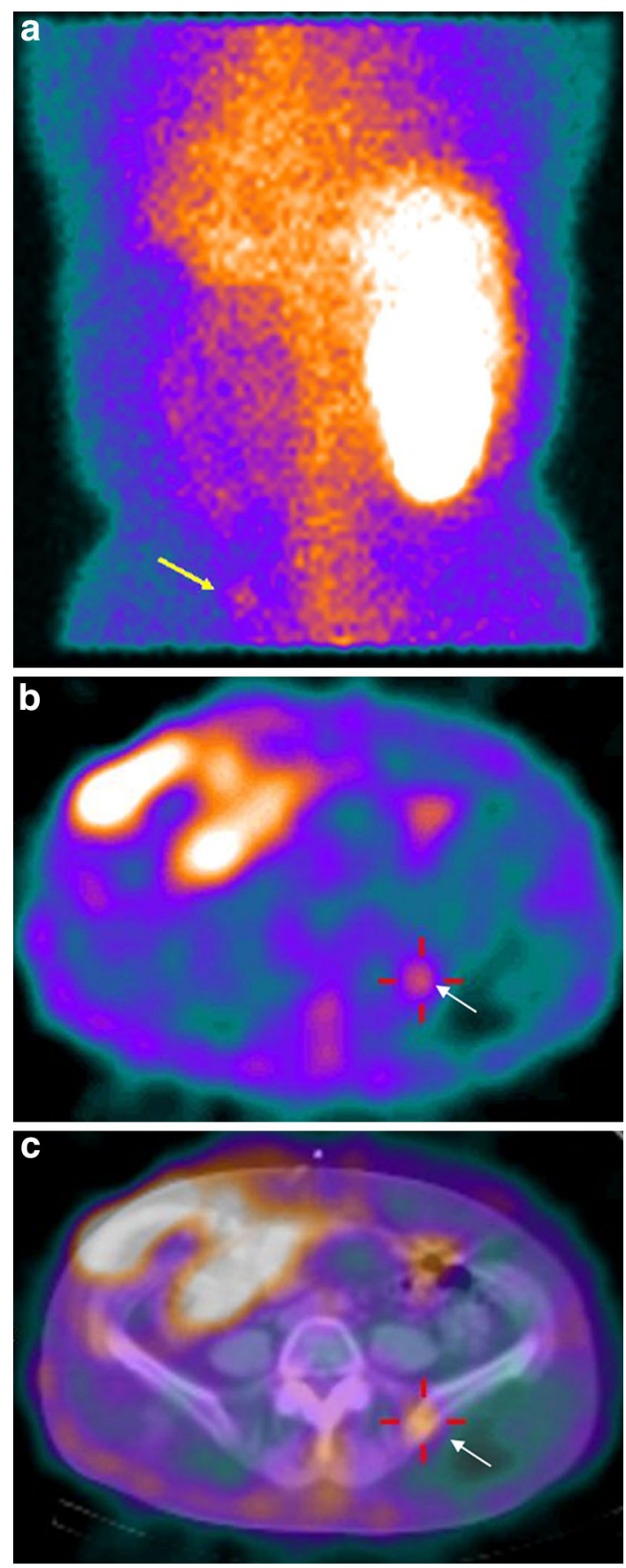

Fig. 1 Planar posterior image (a) from In-111 pentetreotide SRS in a patient with previously resected intestinal carcinoid shows uptake in the left hypogastric-iliac region (arrow). Corresponding transaxial SPECT (b) is not able to precisely localise this uptake (arrow), which SPECT/ CT (c) clearly demonstrates in the left iliac wing (arrow), thus indicating a previously unknown bone metastasis (colour figure online)

SPECT/CT findings caused changes in the therapeutic management of six patients.

The contribution of hybrid SPET/CT imaging to SRS studies and its clinical impact were assessed by Moreira et al. [29] in a group of 12 patients with proven or clinical 



C

Anatonic

Physiologic

Fusion

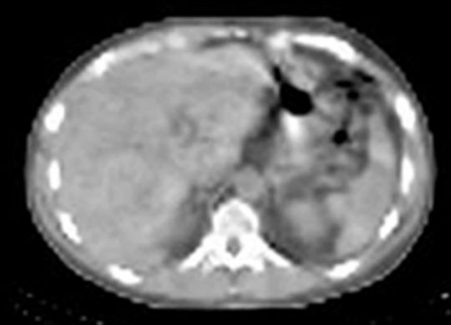

25

Transaxial
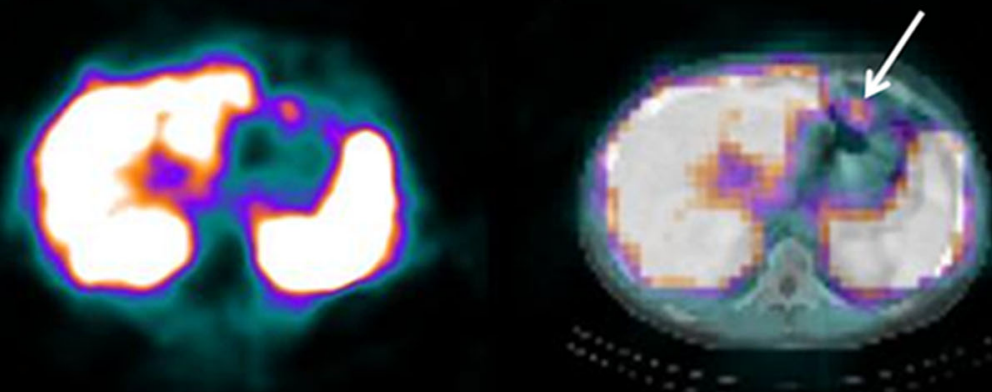

25

Transaxial
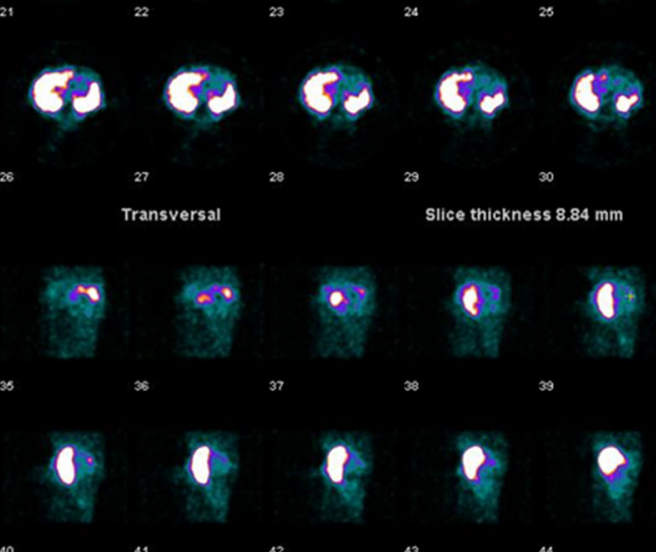

(1)

28

29 Slice thickness $8.84 \mathrm{~mm}$



s.

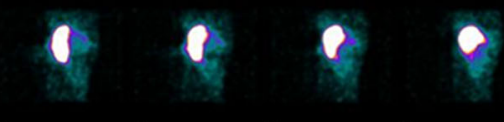

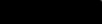

Fig. 2 Planar images (a) from In-111 pentetreotide SRS in a patient with diffuse hepatic involvement from an unknown primary NET. SPECT imaging (b) demonstrates a focus of uptake near the left upper

suspicion of NETs, seven being investigated for staging/ follow-up, and five for primary tumour localisation and staging. SPET/CT data were confirmed through lobe of the liver (see triangulation indicating the hot spot in the different slices), which transaxial fusion SPECT/CT (c) localises in the gastric fornix (arrow) (colour figure online)

comparison with pathological findings, when available, or through clinical and radiological follow-up. Six patients had a negative SRS study and six had positive findings. 

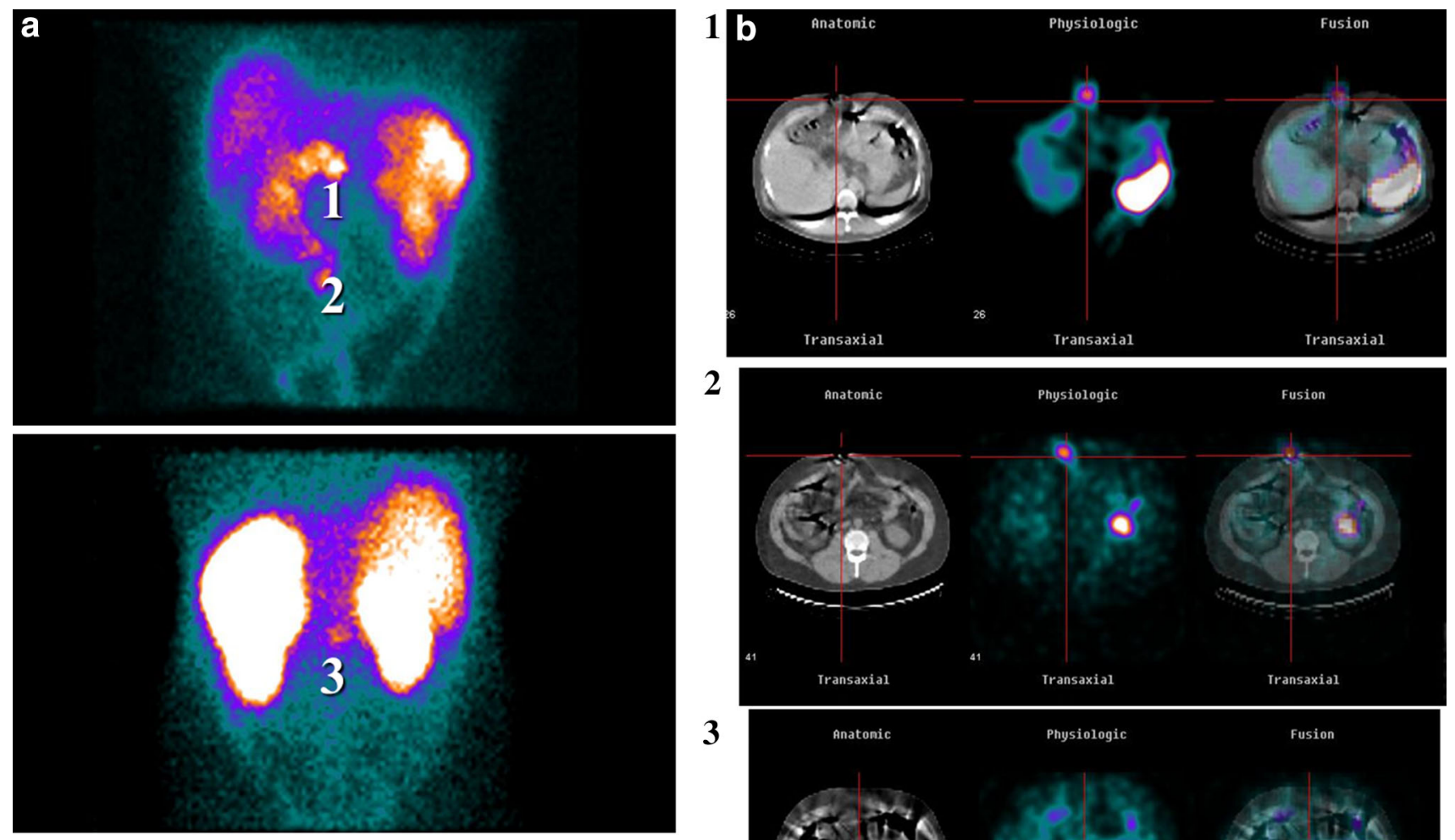

2

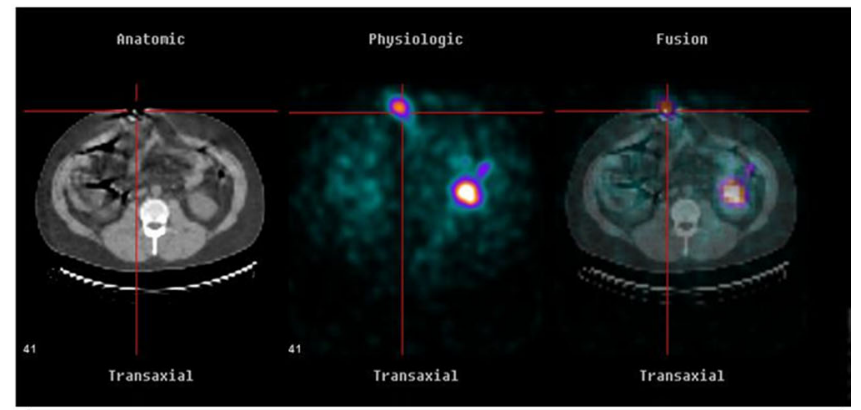

3

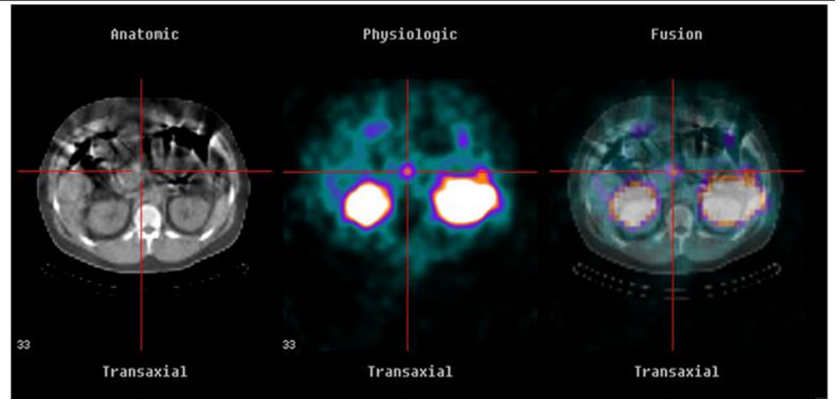

Fig. 3 Planar images (a) from In-111 pentetreotide SRS in a patient with previously resected duodenal gastrinoma demonstrate three foci of abdominal uptake $(1,2$, and 3). Corresponding fused SPECT/CT

SPET/CT improved image interpretation in all six SRSpositive patients, contributing to a better anatomical localisation, and also identified unsuspected bone extension in two patients during follow-up. Moreover, SPET/CT findings affected patient management in three cases. On the basis of these results, the authors concluded that hybrid imaging is an accurate and simple method of registration of functional and anatomical data which enhances the role of SRS in evaluating NETs. SPECT/CT can guide invasive diagnostic and therapeutic procedures and help in monitoring outcomes.

The value of image fusion using SPECT with integrated low-dose CT in comparison with a retrospective voxelbased method of fusion of SPECT and high-resolution CT was evaluated in a series of 27 patients with histologically proven metastatic NETs [30]. The generation of fused images was less time consuming in hybrid SPECT/CT $(<1 \mathrm{~min})$ than in the retrospective image fusion method ( $\sim 25 \mathrm{~min}$ ). Moreover, due to misregistrations in the retrospective fusion of abdominal SPECT, two cases were not

images (b) precisely localise (see triangulation) physiological uptakes within post-surgical abdominal hernias ( 1 and 2$)$ and pathological uptake at a local recurrence (3) (colour figure online)

included in the further analysis. The standard of reference was surgery, biopsy, or imaging during clinical follow-up. For the anatomical assignment of the sites of tracer uptake, SPECT/CT and retrospective fusion revealed overall accuracies of 91 and $94 \%$, respectively. In particular, both image fusion methods were equally well suited for the localisation of SRS foci in parenchymal organs, whereas for the identification of intra-abdominal lymph node metastases, retrospective fusion proved superior, due to the better spatial resolution of diagnostic CT. Nevertheless, the anatomical localisation of SPECT findings using hybrid SPECT/CT was found to be clinically satisfactory, requiring only a small fraction of the time and logistic efforts necessary for the retrospective method.

The clinical impact of SPECT/CT hybrid imaging on SRS studies was retrospectively assessed in 29 patients with known or suspected NETs, to evaluate the extent of disease and thus obtain guidance for the patients' future management [31]. Fifteen out of 29 SPECT-alone studies showed abnormalities. The location of at least one lesion 
was changed or a new location was established as a result of the additional anatomical information from SPECT/CT in 11 of the 15 positive cases $(73 \%)$. In particular, fused images changed the reported anatomical sites of lesions in four of the 11 cases and established previously unknown locations in seven of the 11. Thereafter, confirmation of lesion location was obtained by other investigations during follow-up in seven of these 11 cases (64\%). SPECT/CT affected management in $64 \%$ of the patients in whom additional anatomical information caused a change in the reported location of lesions. The results of this study indicate that a hybrid SPECT/CT system can improve diagnosis and staging of patients with NETs evaluated by SRS with In-111 pentetreotide, thus making it possible to choose more accurately the best therapeutic options.

In a series of 54 patients with known or suspected NETs, the improvement provided by SPECT/CT in the interpretation of SRS SPECT alone and any modification in patient management was retrospectively evaluated [32]. SPECT was negative in nine, positive in 43 , and of uncertain significance in two patients, respectively. In 29 out of 54 patients $(54 \%)$, including eight negative and 21 positive ones, SPECT/CT did not add any significant information to SPECT alone; the two uncertain cases were classified as positive and negative. SPECT/CT images gave additional information in 25 patients ( $46 \%$ ), and improved SPECT interpretation in 23 cases $(42 \%)$, providing better anatomical localisation of increased radiopharmaceutical uptake in 20 cases $(37 \%$ ) and disease exclusion in sites of physiological uptake in five $(9 \%)$. In most cases, the accurate localisation of sites of increased uptake regarded lymph nodes. Moreover, in 10 patients, SPECT/CT allowed definition of the functional significance of CTdetected lesions. The gold standard for the presence of malignancy was either histopathology or clinical or radiological follow-up over at least 6 months. The outcomes of SPECT/CT in this group of patients caused changes in the clinical management in 14 cases, modifying the diagnostic approach in eight and the therapeutic modality in six, respectively.

The more accurate detection and localisation of NETs obtainable with the use of a hybrid SPECT/CT system were subsequently confirmed in a prospective study including 81 patients: in the assessment of these patients, the area under the curve generated by the ROC analysis gave a significantly higher value for SPECT/CT than for SPECT alone [33]. Furthermore, SPECT/CT was able to reduce the number of equivocal cases (i.e. two out of 81 vs 10) and correctly re-classified as probably negative three patients who had been evaluated as positive by SPECT alone. Therefore, SPECT/CT allowed correct classification of a higher number of cases (75 vs 64). Overall, 176 foci of abnormal In-111 pentetreotide uptake were detected by SPECT/CT (172 also by SPECT alone): of these lesions, seven were excluded by subsequent analysis due to a lack of acceptable validation. Thus, the final classification included 138 lesions due to NETs and 31 physiological or benign sites of radiopharmaceutical uptake. The pathological areas were localised in the liver $(n=40)$, lymph nodes $(n=33)$, bone $(n=30)$, lungs $(n=17)$, pancreas $(n=9)$, gut $(n=4)$, thymus $(n=3)$, and soft tissue $(n=2)$. SPECT/CT modified the classification of 25 out of 169 lesions $(14.8 \%)$ : therefore, the global number of correctly classified lesions was 163 out of 169 (96.4\%), with two false-positive results and four equivocal findings. It is worth noting that the fused images dramatically decreased the number of indeterminate results: 4 vs 21 . The number of correctly classified lesions was found to be significantly higher for SPECT/CT, especially in the abdominal area. Finally, taking into account the anatomical localisations of the lesions, SPECT allowed a correct diagnosis in only 77 cases $(45.6 \%$ ), whereas the fused images allowed correct diagnosis in 160 (94.7\%), especially with regard to the lymph nodes, bone, and pancreas. The standard of reference was either histopathology or clinical/imaging follow-up data.

The incremental value of In-111 pentetreotide SPECT/ CT imaging was retrospectively assessed in 49 patients with potential NETs [34]. A total of 89 foci of tracer uptake were detected, involving the head and neck $(n=8)$, chest $(n=11)$, liver $(n=14)$, extrahepatic abdomen $(n=43)$, and bone $(n=13)$, and they were classified according to their location and nature (physiological, benign, or neoplastic). Planar and SPECT images were reviewed by two blinded readers, followed by interpretation using additional SPECT/CT images in a subsequent session, with a third reader providing consensus in cases of disagreement. In no case did planar and SPECT images give a more accurate location than SPECT/CT ones. A consensus read indicated that SPECT/CT provided superior lesion localisation in 55 out of 89 cases $(61.8 \%)$, defined as a more precise interpretation of the anatomical site of radiopharmaceutical uptake. In particular, in 31 out of these 55 lesions (56\%), the improved localisation consisted of uptake in a different organ or an unexpected finding when compared with the results of planar and SPECT-alone images. Moreover, SPECT/CT imaging improved characterisation in 25 of 89 lesions $(28.1 \%)$ and changed their classification. This improved characterisation included the detection of additional sites of disease or inflammation considered significant $(n=12)$, or the demonstration that that lesions initially thought to represent disease were physiological $(n=7)$. These results led to an incremental diagnostic value of SPECT/CT imaging in 20 out of 49 patients (41\%) for reader 1 and 14 out of 49 patients (29\%) for 
reader 2, which was considered likely to affect patient management, in 12 out of $20(60 \%)$ and 7 out of $14(50 \%)$ patients, respectively. Validation of scan interpretation was obtained by histology, CT, MRI or clinical follow-up.

In 24 consecutive patients with suspected or confirmed NETs submitted to In-111 pentetreotide imaging, it was recently evaluated whether SPECT/CT can replace traditional dual time-point planar and SPECT SRS [35]. Planar whole-body images were acquired at 24 and $48 \mathrm{~h}$ postinjection, supplemented with abdominal SPECT/CT imaging at $24 \mathrm{~h}$; chest SPECT/CT was also performed in eight patients at $24 \mathrm{~h}$. Two blinded readers independently evaluated each study. Interpretations were compared with surgical pathology, or clinical and radiological follow-up for at least 12 months. Inter-observer agreement was excellent $(k=0.86)$ for single time-point imaging, and good $(k=0.56)$ for dual time-point imaging on a perlesion basis. In-111 pentetreotide imaging using a single 24-h post-injection time-point with planar and SPECT/CT imaging showed comparable sensitivity (92\%) to planar (24 and $48 \mathrm{~h}$ ) and SPECT (24 h) dual time-point imaging (83\%), maintaining excellent specificity (100\%). These results indicate that SPECT/CT improves reader confidence in confirming normal biodistribution of In-111 pentetreotide, and distinguishing between abdominal tumour and physiological activity. Routine use of SPECT/ $\mathrm{CT}$ in addition to planar imaging at $24 \mathrm{~h}$ allows single time-point imaging, making SRS more comfortable for the patient, and restricting the acquisition of delayed images to selected cases.

SPECT/CT allows not only image fusion but also attenuation correction (AC) of SPECT data. The impact of AC on SRS SPECT data in patients examined by hybrid SPECT/CT has been evaluated in two studies. In the first one, 17 patients with NETs were considered: the intensity and contrast of 46 foci classified as pathological were rated in both the non-attenuation corrected (NAC) and the AC SPECT images [36]. Focus contrast and intensity significantly increased in 14 out of 46 foci $(30 \%)$ after AC in the visual analysis, with increasing focus depth. These findings indicate that AC SRS SPECT imaging performed by means of a hybrid SPECT/CT system causes more clearly contrasted foci in visual analysis, especially in the more centrally localised sites of tracer uptake. Therefore, CT-based AC has the potential to improve the sensitivity of SRS SPECT, but this aspect could not be assessed by this study because the great majority of the foci examined already showed high intensity in the NAC SPECT images.

The impact of AC by low-dose CT with a hybrid SPECT/CT device on SRS sensitivity was specifically addressed by Steffen et al. [37] in a retrospective analysis of 50 consecutive patients with NETs. All the sites of uptake on both the NAC and AC SPECT images were scored for intensity and contrast using a six-point scale. Final verification of lesions was based on CT/MRI data and/or clinical follow-up. Of 227 foci, 222 (98\%) were visible in both the NAC and the AC SPECT images, whereas five foci (liver, $n=2$; bone, $n=2$; lymph node, $n=1)$ in three patients were detected only in the AC SPECT images. These results led to a $2 \%$ increase in sensitivity but without any change in the therapeutic decisions. Moreover, in 67 out of 227 foci (29\%), focus intensity/contrast increased after AC, with only five lesions showing a decrease, suggesting that AC of SRS SPECT significantly improves focus visualisation, but only slightly increases sensitivity.

The impact of SPECT/CT on inter-rater agreement in the interpretation of SRS findings has been retrospectively studied in a series of 25 unselected patients with suspected or histologically proven NETs [38]. SRS was independently interpreted by two nuclear medicine physicians: an experienced one and an inexperienced one. Both readers first re-evaluated the planar whole-body images alone, then added the SPECT images, and finally the CT images. Lesions with increased radiopharmaceutical uptake were classified as equivocal, probably pathological, and definitely pathological. Globally, 50 lesions were found in 23 patients. The two readers showed only moderate agreement in the interpretation of the planar findings, with an improvement obtained by adding SPECT and very good agreement by adding SPECT/CT. Evaluation of hybrid images led to up-staging of $18 \%$ of the lesions and downstaging of $12 \%$ compared to planar plus SPECT (experienced reader), and reduced the frequency of indefinite scores (equivocal, probably pathological) from $18 \%$ in planar plus SPECT to $6 \%$. In particular, the change in lesion localisation with SPECT/CT tended to contribute to modifying the lesion classification. These outcomes indicate that low-dose SPECT/CT stabilises report quality in SRS, improving image interpretation by increasing interrater agreement in patients with NETs. Therefore, hybrid images help in the interpretation of SRS, reducing the gap in performance between inexperienced and experienced physicians.

The impact on patient management is a fundamental index when assessing the clinical value of any imaging method. In this regard, the above-mentioned SPECT/CT results are summarised in Table 1 . Globally, hybrid imaging changed the management in $25 \%$ of NET patients (i.e. 71 out of 285) evaluated with In-111 pentetreotide imaging.

\section{Radioidinated MIBG SPECT/CT}

Apart from the previously cited papers that included patients evaluated also with MIBG [26, 28], the value of 
SPECT/CT in I-123 MIBG imaging was first investigated as a means of avoiding false-positive planar scintigraphy interpretations in 31 patients with suspected pheochromocytoma who had focal radiopharmaceutical uptake detected on planar scans [39]. Planar and SPECT images were initially evaluated by two nuclear medicine physicians and then compared with SPECT/CT findings with the help of a radiologist. In 25 cases $(81 \%)$ the focal uptake visible on planar images was clarified by hybrid imaging as physiological activity (Fig. 4); in the remaining six patients, SPECT/CT helped in identifying the pathological sites of uptake. Validation of scan interpretation was obtained by histology, CT, MRI or clinical follow-up.

Table 1 Main studies reporting the clinical impact of SPECT/CT with In-111 pentetreotide in NETs

\begin{tabular}{llc}
\hline Authors & $\begin{array}{l}\text { No. of } \\
\text { patients }\end{array}$ & $\begin{array}{l}\text { Change of } \\
\text { management (\%) }\end{array}$ \\
\hline Krausz et al. [27] & 72 & $10 / 72(14)$ \\
Pfannenberg et al. [28] & 54 & $15 / 54(28)$ \\
Schillaci [24] & 19 & $6 / 19(32)$ \\
Moreira et al. [29] & 12 & $3 / 12(25)$ \\
Hillel et al. [31] & 29 & $7 / 29(24)$ \\
Castaldi et al. [32] & 50 & $14 / 50(28)$ \\
Wong et al. [34] & 49 &
\end{tabular}

* At least one major change for one reader
The usefulness of MIBG SPECT/CT for the diagnosis of pheochromocytoma was analysed in a retrospective study including 22 patients (four evaluated with I-131 MIBG and 18 with I-123 MIBG) [40]. In 14 cases, MIBG SPECT/CT was positive and correlated with CT or MRI: six adrenal, four extra-adrenal, three metastatic disease, and one recurrent lesion. Of these patients, 10 had pheochromocytoma confirmed by histology after surgical resection, three were known to have metastatic pheochromocytoma, and one was managed conservatively with close clinical follow-up. Diagnostic confidence and precision of anatomical localisation of pheochromocytoma were improved by SPECT/CT in six cases and the original radiological diagnosis was revised in three patients. It is worth noting that four patients with a pheochromocytoma-associated germline mutation had multifocal disease excluded by SPECT/CT, whereas patients who had positive biochemistry and a solitary lesion on conventional imaging without a germline mutation obtained no diagnostic improvement by hybrid imaging. In eight cases MIBG SPECT/CT was negative: in these cases the diagnostic confidence for exclusion of a pheochromocytoma was improved in five (including one patient with a germline mutation), and the original radiological diagnosis was changed in three, including one false-positive planar MIBG result. The gold standard for the diagnosis of a pheochromocytoma was histology; a negative finding was confirmed by repeated


Fig. 4 I-123 MIBG scintigraphy in a patient with clinical suspicion of pheochromocytoma. Planar images (a) show an area of increased focal uptake in the right upper abdomen (arrow in the posterior scan), which SPECT/CT (b) is able to clearly localise (see triangulation) within the right kidney (i.e. physiological renal excretion) (colour figure online) 
normal biochemistry. The results of this study indicate that MIBG SPECT/CT is able to improve the overall assessment of pheochromocytoma by increasing diagnostic confidence; clinically, hybrid imaging was shown to be particularly useful in patients at high risk of pheochromocytoma (with a pheochromocytoma-associated germline mutation or previous history of pheochromocytoma or paraganglioma), for the confirmation of small extra-adrenal pheochromocytomas, small metastatic lesions or recurrence at previous surgical sites.

In a retrospective institutional study of the use of I-131MIBG SPECT/CT in NETs, 28 patients showed equivocal findings on planar imaging [41]. The contribution of hybrid SPECT/CT imaging in these studies was analysed, and in 27 out of 28 cases, it provided vital additional information. In particular, SPECT/CT was useful in distinguishing physiological liver and bowel activities from tumour uptake, and helped in localising primary as well as metastatic NETs.

The incremental value of I-131 MIBG SPECT/CT over planar images and SPECT alone and its usefulness in overcoming the limitations associated with poor imaging properties of I-131 MIBG were recently retrospectively assessed in 63 patients with clinical or biochemical suspicion of pheochromocytoma [42]. The final diagnosis (based on postoperative histopathological findings or clinical/ imaging follow-up) showed that 30 out of 126 adrenals in 28 patients had pheochromocytoma: 26 patients had unilateral and two had bilateral lesions. The scintigraphic images, of all kinds, were independently evaluated by two nuclear medicine physicians with different experience (reader number 1: 6 years: reader number 2: 2 years). According to reader number 1 , on planar scans, 19 adrenals were true positive, 96 were true negative, none was false positive, and 11 were false negative; according to reader number 2, 12 were true positive, 94 were true negative, two were false positive, and 18 were false negative. On SPECT imaging, for reader number 1,26 adrenals were true positive, 93 were true negative, three were false positive, and four were false negative; for reader number 2, 26 adrenals were true positive, 83 were true negative, 13 were false positive, and four were false negative. On hybrid SPECT/CT images, for reader number 1, 27 adrenals were true positive, 96 were true negative, none was false positive, and three were false negative; for reader number 2, 27 adrenals were true positive, 96 were true negative, none was false positive, and three were false negative. The results of receiver operating characteristic curve analysis with the areas under the curves indicated that, for both readers, the diagnostic accuracy of planar images was significantly lower than that of SPECT and SPECT/CT, and the accuracy of SPECT was significantly lower than that of SPECT/CT. The findings of this study suggest that
I-131MIBG SPECT/CT is more accurate than planar scintigraphy and SPECT alone in characterising adrenal lesions in patients with clinical or biochemical suspicion of pheochromocytoma; moreover, hybrid imaging is able to improve observer confidence and inter-observer agreement.

Franzius et al. compared I-123 MIBG SPECT/CT to the C-11 labelled radiopharmaceutical metahydroxyephedrine (HED), a noradrenaline analogue specifically developed for PET imaging of the sympathetic nervous system, whose uptake reflects catecholamine transport, storage, as well as catecholamine recycling [43]. Twenty-four pairs of examinations were performed in 19 patients with known or suspected tumours of the sympathetic nervous system. The intensity of radiopharmaceutical uptake above background was visually analysed on both PET and SPECT images using a four-point scale. In five patients clinical follow-up and/or histological examination did not reveal any tumour deriving from the sympathetic nervous system; in the remaining 14 patients: six neuroblastomas, five pheochromocytomas, two paragangliomas, and one ganglioneuroblastoma were histologically confirmed. There were no false-positive results either with MIBG or HED. PET/CT detected 80 out of 81 tumour lesions (soft tissue 61; bone, 19), whereas SPECT/CT visualised 75 out of 81 lesions (soft tissue 56; bone, 19). The tumour-to-background contrast of C-11 HED accumulation was higher compared with I-123 MIBG uptake in 26 lesions, equal in 39 , and lower in 16.

Subsequently, a retrospective study was performed to assess and compare F-18 L-dihydroxyphenylalanine (DOPA) PET/CT and I-123 MIBG SPECT/CT in the restaging of 12 patients with known or suspected recurrent tumour after initial surgery for pheochromocytoma or extra-adrenal paraganglioma; cytohistology and a combination of laboratory and imaging studies and follow-up were used as reference standard [44]. F-18 DOPA is a radiopharmaceutical useful for PET imaging of a wide range of tumours, including NETs and catecholaminesecreting tumours $[3,45]$. In this series, even though the difference was not statistically significant on per-patient analysis, F-18 DOPA provided better results than I-123 MIBG SPECT/CT: PET/CT was positive in all patients, whereas SPECT/CT had three false-negative results. These findings indicate a superiority of F-18 DOPA PET/CT over I-123 MIBG SPECT/CT in evaluating disease extension in patients with recurrent paraganglioma; nevertheless, in patients with inoperable disease, only MIBG imaging allows the selection of patients suitable for I-131 MIBG therapy.

The contribution of I-123 MIBG SPECT/CT to contrastenhanced CT image analysis in the follow-up of patients with neuroblastoma and pheochromocytoma and its impact on patient management were retrospectively assessed in a 
group of 11 cases, eight children with neuroblastoma, and three adults with pheochromocytoma [46]. Clinical followup was taken as the gold standard. Overall, of 15 imaging studies, SPECT/CT provided additional information in eight out of 15 cases (53\%) and in particular in eight out of nine discordant studies $(89 \%)$ between SPECT/CT and diagnostic CT. The findings of this study indicated that in cases of equivocal diagnostic CT (mainly distorted anatomy) or of suboptimal localisation of the foci of MIBG uptake, SPECT/CT is very useful clinically to define the anatomical location of these foci and to characterise the significance (benign or malignant) of uncertain CT findings. It is worth noting that the low-resolution CT images of SPECT/CT could be valuably incremented by the superior diagnostic contrast-enhanced CT.

Fukuoka et al. [47] retrospectively evaluated 16 patients with malignant pheochromocytoma/paraganglioma and neuroblastoma, who were referred for I-131 MIBG therapy, with the aims of comparing lesion detectability of I-123 MIBG scintigraphy with that of high-dose post-therapy I-131 MIBG and of assessing the incremental benefit of SPECT/CT over planar scans for the detection and localisation of the lesions. Thirty-one studies with I-123 MIBG were acquired in 16 patients and 17 studies of high-dose I-131 MIBG were performed in 12 patients: planar and SPECT/CT images were compared for lesion detectability and localisation. Moreover, the lesion detectability in 10 pairs of I-123 and high-dose I-131 MIBG studies of the same patient, acquired within 2 weeks, were evaluated. In I-123 MIBG imaging, a total of 145 and 155 sites of pathological uptake were detected by planar and hybrid imaging, respectively; in post-therapy I-131 MIBG scintigraphy, a total of 136 and 140 abnormal foci were detected by planar and SPECT/CT imaging, respectively. Hybrid imaging provided additional diagnostic information over planar scans in 25 studies $(81 \%)$ of 12 patients (75\%) with I-123 MIBG and in nine studies (53\%) of nine patients $(75 \%)$ with high-dose I-131 MIBG. It is worth noting that most of the lesions detected only on SPECT/CT were located near the physiological uptake or overlapped with the physiological uptake. In the 10 pairs of I-123 and high-dose I-131 MIBG studies in the same patients, the number of lesions visualised by I-123 MIBG planar scans and SPECT/CT and post-therapy planar I-131 MIBG and SPECT/CT were 3.0 and 3.7, 7.3 and 7.7 per study, respectively.

A recent paper evaluated the diagnostic performance of I-123 MIBG SPECT/MRI, I-123 MIBG SPECT/CT, and MRI for the detection of adrenal pheochromocytomas in patients with elevated catecholamines [48]. Coregistration and fusion of SPECT/CT and adrenal MRI examinations were undertaken on a workstation with a commercial software package using the SPECT and the corresponding
CT series as reference. Sixteen adrenal tumours were found in 13 patients. Histopathology and/or clinical and radiological follow-up served as the gold standard. On a perlesion basis, SPECT/CT had a sensitivity of $87.5 \%$, a specificity of $94 \%$, and an accuracy of $92.5 \%$; MRI had a sensitivity of $87.5 \%$, a specificity of $97 \%$, and an accuracy of $95 \%$. On a per-patient basis, both SPECT/CT and MRI had a sensitivity of $86 \%$, a specificity of $93 \%$, and an accuracy of $91 \%$. SPECT/MRI fusion was superior to both SPECT/CT and MRI and had a sensitivity of $100 \%$ on both a per-lesion and a per-patient basis. This study demonstrated the feasibility and diagnostic performance of retrospective I-123 MIBG SPECT/MRI (based on hybrid SPECT/CT), which was found to be the most accurate imaging modality for detecting pheochromocytoma.

Finally, the improved benefit of SPECT/CT compared to SPECT alone for the accurate localisation of NETs was recently confirmed in a study including five I-123 MIBG scans and eight SRS studies [49]. Pathology and radiological follow-up served as the reference standard. In MIBG scans, SPECT alone could not detect a $1.1-\mathrm{cm}$ adrenal lesion or correctly characterise normal physiological adrenal uptake in consecutive scans of the same patient with a prior history of adrenelectomy, all of which were accurately assessed by means of SPECT/CT. In In-111 pentetreotide studies, SPECT/CT performed better than SPECT alone for lesion localisation and characterisation in the liver, pancreatic head, bones, and lymph nodes. In fact, SPECT alone could not localise primary or metastatic lesions in six subjects all of which were accurately identified by SPECT/CT. These results indicate that SPECT/CT is clinically useful in imaging patients with NETs by improving lesion conspicuity, reducing false positives, and clarifying indeterminate lesions due to a better localisation capability compared to SPECT alone; therefore, it should be included in the diagnostic work-up of these patients rather than SPECT alone.

\section{Future perspectives}

During the last decade, hybrid imaging has revolutionised nuclear medicine: multimodal devices integrating PET or SPECT with CT are now able to combine functional and anatomical data in the same diagnostic examination in everyday clinical practice. The increasing availability of hybrid SPECT/CT devices offers superior accuracy for localisation and functional characterisation of NETs in comparison with traditional planar and SPECT-alone images. Nevertheless, in our opinion, it is of the utmost importance that the imaging protocol be tailored to each patient. In particular, performing CT should be fully justified and the CT procedure should be individually adjusted 
tailored to address the clinical issue. To date, low-resolution CT has usually been performed in SPECT/CT examinations [50], but clearly it does not always allow optimal interpretation of the CT images, which could provide superior anatomical resolution if acquired by a full diagnostic contrast-enhanced protocol. Therefore, new-generation SPECT/CT hybrid cameras with improved CT spatial resolution will enable the avoidance of an additional, separate contrast-enhanced CT study in clinical situations that demand superior resolution.

Because SPECT images are coarsely compromised by artefacts caused by photon scatter and attenuation, current SPECT cameras do not allow the quantification of regional values of radioactivity tissue concentration. Hybrid SPECT/CT systems seem to be able to provide reliable corrections for these artefacts, thereby allowing truly quantitative SPECT [51, 52]. Therefore, the use of these devices has the potential to substantially improve the accuracy of dosimetry estimates in radionuclide therapy for NETs [53-55].

However, the real question for the future clinical applications of SPECT/CT in patients with NETs is related to the advent of PET/CT. In particular, recent years have seen Ga-68 DOTA-labelled somatostatin analogues for PET imaging introduced for the diagnostic work-up of NETs with very interesting results; the accuracy is usually higher than that of In-111 pentetreotide imaging in comparative studies $[56,57]$. It is now evident that nuclear medicine departments with PET/CT facilities are moving towards PET applications, with In-111 pentetreotide and MIBG SPECT/CT being considered a second-choice approach for NET diagnosis, staging, and follow-up, except in centres where PET/CT or some PET radiopharmaceuticals are still not available.

\section{Conclusions}

SPECT/CT is a hybrid imaging technique, which combines the benefits of the functional information from SPECT with the detailed anatomical data provided by CT. Several studies analysed in this review have clearly indicated that, in patients with NETs, SPECT/CT is able to improve the diagnostic accuracy of planar and SPECT-alone images, using either In-111 pentetreotide or radioidinated MIBG. Apart from the advantages of better sensitivity and specificity, SPECT/CT changes the clinical management in a significant percentage of patients with NETs and should therefore always be performed. In-111 pentetreotide and MIBG with hybrid system SPECT/CT are, for NETs, currently the best available imaging options with gammaemitting radiopharmaceuticals, and this approach can still be considered a very useful alternative to PET/CT. The role of SPECT/CT in the future routine clinical scenario will mainly depend on the large-scale availability of the PET radiopharmaceuticals for NET imaging.

Conflict of interest All the authors of the manuscript (i.e. Orazio Schilllaci, Angela Spanu, Barbara Palumbo, and Roberta Danieli) declare no conflict of interest.

Human and animal studies This article does not contain any studies with human or animal subjects performed by any of the authors.

\section{References}

1. Koopmans KP, Neels ON, Kema IP, Elsinga PH, Links TP, de Vries EG, Jager PL (2009) Molecular imaging in neuroendocrine tumors: molecular uptake mechanisms and clinical results. Crit Rev Oncol Hematol 71:199-213. doi:10.1016/j.critrevonc.2009. 02.009

2. Yao JC, Hassan M, Phan A, Dagohoy C, Leary C, Mares JE, Abdalla EK, Fleming JB, Vauthey JN, Rashid A, Evans DB (2008) One hundred years after "carcinoid": epidemiology of and prognostic factors for neuroendocrine tumors in 35,825 cases in the United States. J Clin Oncol 26:3063-3072. doi:10.1200/JCO. 2007.15.4377

3. Schillaci O (2014) ${ }^{18}$ F-DOPA and other radiopharmaceuticals for imaging unknown primary neuroendocrine tumors. J Nucl Med 55:357-359. doi:10.2967/jnumed.113.133116

4. Oberg K (2000) State of the art and future prospects in the management of neuroendocrine tumors. Q J Nucl Med 44:3-12

5. Sharma P, Singh H, Bal C, Kumar R (2014) PET/CT imaging of neuroendocrine tumors with (68)Gallium-labeled somatostatin analogues: an overview and single institutional experience from India. Indian J Nucl Med 29:2-12. doi:10.4103/0972-3919. 125760

6. Klimstra DS, Modlin IR, Coppola D, Lloyd RV, Suster S (2010) The pathologic classification of neuroendocrine tumors: a review of nomenclature, grading, and staging systems. Pancreas 39:707-712

7. van Essen M, Sundin A, Krenning EP, Kwekkeboom DJ (2014) Neuroendocrine tumours: the role of imaging for diagnosis and therapy. Nat Rev Endocrinol 10:102-114. doi:10.1038/nrendo. 2013.246

8. Volterrani D, Orsini F, Chiacchio S (2013) Multiagent targeting of neuroendocrine neoplasms. Clin Transl Imaging 1:407-421. doi:10.1007/s40336-013-0043-x

9. Bison SM, Konijnenberg MW, Melis M, Pool SE, Bernsen MR, Teunissen JJ, Kwekkeboom DJ, de Jong M (2014) Peptide receptor radionuclide therapy using radiolabeled somatostatin analogs: focus on future developments. Clin Transl Imaging 2:55-66. doi:10.1007/s40336-014-0054-2

10. Lamberts SWJ, Bakker WH, Reubi JC, Krenning EP (1990) Somatostatin-receptor imaging in the localization of endocrine tumors. N Engl J Med 323:1246-1249

11. Sisson JC, Yanik GA (2012) Theranostics: evolution of the radiopharmaceutical meta-iodobenzylguanidine in endocrine tumors. Semin Nucl Med 42:171-184. doi:10.1053/j.sem nuclmed.2011.11.004

12. Kuwert T, Schillaci O (2014) SPECT/CT: yesterday, today, tomorrow. Clin Transl Imaging. doi:10.1007/s40336-014-0088-5

13. Toumpanakis C, Kim MK, Rinke A, Bergestuen DS, Thirlwell C, Khan MS, Salazar R, Oberg K (2014) Combination of crosssectional and molecular imaging studies in the localization of 
gastroenteropancreatic neuroendocrine tumors. Neuroendocrinology 99:63-74. doi:10.1159/000358727

14. Krenning EP, Kwekkeboom DJ, Bakker WH et al (1993) Somatostatin receptor scintigraphy with $\left[{ }^{111} \mathrm{In}-\mathrm{DTPA}-\mathrm{D}-\mathrm{Ph} \mathrm{e}^{1}\right]$ and $\left[{ }^{123} \mathrm{I}-\mathrm{Tyr}^{3}\right]$-octreotide: the Rotterdam experience with more than 1000 patients. Eur J Nucl Med 20:716-731

15. Termanini B, Gibril F, Reynolds JC et al (1997) Value of somatostatin receptor scintigraphy: a prospective study in gastrinoma of its effect on clinical management. Gastroenterology 112:335-347

16. Corleto VD, Scopinaro F, Angeletti S, Materia A, Basso N, Polettini E, Annibale B, Schillaci O, D’Ambra G, Marignani M, Gualdi G, Bordi C, Passaro EJ, Delle Fave G (1996) Somatostatin receptor localization of pancreatic endocrine tumors. World $\mathbf{J}$ Surg 20:241-244

17. Schillaci O, Spanu A, Scopinaro F et al (2003) Somatostatin receptor scintigraphy in liver metastasis detection from gastroenteropancreatic neuroendocrine tumors. J Nucl Med 44:359-368

18. Gibril F, Jensen RT (2004) Diagnostic uses of radiolabelled somatostatin receptor analogues in gastroenteropancreatic endocrine tumours. Dig Liver Dis 36(Suppl 1):S106-S120

19. Schillaci O, Corleto VD, Annibale B et al (1999) Single photon emission computed tomography procedure improves accuracy of somatostatin receptor scintigraphy in gastroenteropancreatic tumours. Ital J Gastroenterol Hepatol 31(Suppl 2):S186-S189

20. Schillaci O, Scopinaro F, Angeletti S et al (1996) SPECT improves accuracy of somatostatin receptor scintigraphy in abdominal carcinoid tumors. J Nucl Med 37:1452-1456

21. Schillaci O, Filippi L, Danieli R, Simonetti G (2007) Singlephoton emission computed tomography/computed tomography in abdominal diseases. Semin Nucl Med 37:48-61

22. Pérault C, Schvartz C, Wampach H, Liehn JC, Delisle MJ (1997) Thoracic and abdominal SPECT-CT image fusion without external markers in endocrine carcinomas: the group of thyroid tumoral pathology of champagne-ardenne. J Nucl Med 38:1234-1242

23. Fujita A, Hyodoh H, Kawamura Y, Kanegae K, Furuse M, Kanazawa K (2000) Use of fusion images of I-131 metaiodobenzylguanidine, SPECT, and magnetic resonance studies to identify a malignant pheochromocytoma. Clin Nucl Med $25: 440-442$

24. Schillaci O (2004) Functional-anatomical image fusion in neuroendocrine tumors. Cancer Biother Radiopharm 19:129-134

25. Thierens HM, Monsieurs MA, Bacher K (2005) Patient dosimetry in radionuclide therapy: the whys and the wherefores. Nucl Med Commun 26:593-599

26. Even-Sapir E, Keidar Z, Sachs J et al (2001) The new technology of combined transmission and emission tomography in evaluation of endocrine neoplasms. J Nucl Med 42:998-1004

27. Krausz Y, Keidar Z, Kogan I, Even-Sapir E, Bar-Shalom R, Engel A, Rubinstein R, Sachs J, Bocher M, Agranovicz S, Chisin $\mathrm{R}$, Israel O (2003) SPECT/CT hybrid imaging with ${ }^{111}$ In-pentetreotide in assessment of neuroendocrine tumours. Clin Endocrinol (Oxf) 59:565-573

28. Pfannenberg AC, Eschmann SM, Horger M et al (2003) Benefit of anatomical-functional image fusion in the diagnostic work-up of neuroendocrine neoplasms. Eur J Nucl Med Mol Imaging 30:835-843

29. Moreira AP, Duarte LH, Vieira F, João F, Lima JP (2005) Value of SPET/CT image fusion in the assessment of neuroendocrine tumours with ${ }^{111}$ In-pentetreotide scintigraphy. Rev Esp Med Nucl 24:14-18

30. Amthauer H, Denecke T, Rohlfing T, Ruf J, Böhmig M, Gutberlet M, Plöckinger U, Felix R, Lemke AJ (2005) Value of image fusion using single photon emission computed tomography with integrated low dose computed tomography in comparison with a retrospective voxel-based method in neuroendocrine tumours. Eur Radiol 15:1456-1462

31. Hillel PG, van Beek EJ, Taylor C, Lorenz E, Bax ND, Prakash V, Tindale WB (2006) The clinical impact of a combined gamma camera/CT imaging system on somatostatin receptor imaging of neuroendocrine tumours. Clin Radiol 61:579-587

32. Castaldi P, Rufini V, Treglia G, Bruno I, Perotti G, Stifano G, Barbaro B, Giordano A (2008) Impact of ${ }^{111}$ In-DTPA-octreotide SPECT/CT fusion images in the management of neuroendocrine tumours. Radiol Med 113:1056-1067. doi:10.1007/s11547-0080319-9

33. Perri M, Erba P, Volterrani D, Lazzeri E, Boni G, Grosso M, Mariani G (2008) Octreo-SPECT/CT imaging for accurate detection and localization of suspected neuroendocrine tumors. Q J Nucl Med Mol Imaging 52:323-333

34. Wong KK, Cahill JM, Frey KA, Avram AM (2010) Incremental value of ${ }^{111}$-in pentetreotide SPECT/CT fusion imaging of neuroendocrine tumors. Acad Radiol 17:291-297. doi:10.1016/j.acra. 2009.08.015

35. Wong KK, Wynn EA, Myles J, Ackermann RJ, Frey KA, Avram AM (2011) Comparison of single time-point [ ${ }^{111}$-In] pentetreotide SPECT/CT with dual time-point imaging of neuroendocrine tumors. Clin Nucl Med 36:25-31. doi:10.1097/ RLU.0b013e3181feedc0

36. Ruf J, Steffen I, Mehl S, Rosner C, Denecke T, Pape UF, Plotkin M, Amthauer H (2007) Influence of attenuation correction by integrated low-dose CT on somatostatin receptor SPECT. Nucl Med Commun 28:782-788

37. Steffen IG, Mehl S, Heuck F, Elgeti F, Furth C, Amthauer H, Ruf J (2009) Attenuation correction of somatostatin receptor SPECT by integrated low-dose CT: is there an impact on sensitivity? Clin Nucl Med 34:869-873. doi:10.1097/RLU.0b013e3181becfcb

38. Apostolova I, Riethdorf S, Buchert R, Derlin T, Brenner W, Mester J, Klutmann S (2010) SPECT/CT stabilizes the interpretation of somatostatin receptor scintigraphy findings: a retrospective analysis of inter-rater agreement. Ann Nucl Med 24:477-483. doi:10.1007/s12149-010-0383-9

39. Ozer S, Dobrozemsky G, Kienast O, Beheshti M, Becherer A, Niederle B, Kainberger F, Dudczak R, Kurtaran A (2004) Value of combined XCT/SPECT technology for avoiding false positive planar $\left({ }^{123}\right)$ I-MIBG scintigraphy. Nuklearmedizin 43:164-170

40. Meyer-Rochow GY, Schembri GP, Benn DE, Sywak MS, Delbridge LW, Robinson BG, Roach PJ, Sidhu SB (2010) The utility of metaiodobenzylguanidine single photon emission computed tomography/computed tomography (MIBG SPECT/CT) for the diagnosis of pheochromocytoma. Ann Surg Oncol 17:392-400. doi:10.1245/s10434-009-0850-5

41. Verma P, Chanadana JH, Shanthly N, Oommen R (2012) Iodine131MIBG SPECT/CT in neuroendocrine tumours: an institutional experience. Indian J Nucl Med 27:246-248. doi:10.4103/ 0972-3919.115396

42. Sharma P, Dhull VS, Jeph S, Reddy RM, Singh H, Naswa N, Bal C, Kumar R (2013) Can hybrid SPECT-CT overcome the limitations associated with poor imaging properties of ${ }^{131} \mathrm{I}-\mathrm{MIBG}$ ?: comparison with planar scintigraphy and SPECT in pheochromocytoma. Clin Nucl Med 38:e346-e353. doi:10.1097/RLU. 0b013e318279bcb2

43. Franzius C, Hermann K, Weckesser M, Kopka K, Juergens KU, Vormoor J, Schober O (2006) Whole-body PET/CT with ${ }^{11} \mathrm{C}$ meta-hydroxyephedrine in tumors of the sympathetic nervous system: feasibility study and comparison with ${ }^{123}$ I-MIBG SPECT/CT. J Nucl Med 47:1635-1642

44. Rufini V, Treglia G, Castaldi P, Perotti G, Calcagni ML, Corsello SM, Galli G, Fanti S, Giordano A (2011) Comparison of ${ }^{123}$ IMIBG SPECT-CT and ${ }^{18}$ F-DOPA PET-CT in the evaluation of patients with known or suspected recurrent paraganglioma. Nucl 
Med Commun 32:575-582. doi:10.1097/MNM. 0b013e328345a340

45. Calabria F, Chiaravalloti A, Di Pietro B, Grasso C, Schillaci O (2012) Molecular imaging of brain tumors with ${ }^{18}$ F-DOPA PET and PET/CT. Nucl Med Commun 33:563-570. doi:10.1097/ MNM.0b013e328351d566

46. Rozovsky K, Koplewitz BZ, Krausz Y, Revel-Vilk S, Weintraub M, Chisin R, Klein M (2008) Added value of SPECT/CT for correlation of MIBG scintigraphy and diagnostic CT in neuroblastoma and pheochromocytoma. AJR Am J Roentgenol 190:1085-1090. doi:10.2214/AJR.07.2107

47. Fukuoka M, Taki J, Mochizuki T, Kinuya S (2011) Comparison of diagnostic value of I-123 MIBG and high-dose I-131 MIBG scintigraphy including incremental value of SPECT/CT over planar image in patients with malignant pheochromocytoma/ paraganglioma and neuroblastoma. Clin Nucl Med 36:1-7. doi:10.1097/RLU.0b013e3181feeb5e

48. Derlin T, Busch JD, Wisotzki C, Schoennagel BP, Bannas P, Papp L, Klutmann S, Habermann CR (2013) Intraindividual comparison of ${ }^{123} \mathrm{I}$-mIBG SPECT/MRI, ${ }^{123} \mathrm{I}$-mIBG SPECT/CT, and MRI for the detection of adrenal pheochromocytoma in patients with elevated urine or plasma catecholamines. Clin Nucl Med 38:e1-e6. doi:10.1097/RLU.0b013e318263923d

49. Bural GG, Muthukrishnan A, Oborski MJ, Mountz JM (2012) Improved benefit of SPECT/CT compared to SPECT alone for the accurate localization of endocrine and neuroendocrine tumors. Mol Imaging Radionucl Ther 21:91-96. doi:10.4274/ Mirt.80299

50. Bach-Gansmo T, Schwarzlmüller T, Jøraholmen V, Salbu J, Biermann M, Naum A, Kleven-Madsen N (2010) SPECT/CT hybrid imaging; with which CT? Contrast Media Mol Imaging 5:208-212. doi:10.1002/cmmi.375

51. Ritt P, Kuwert T (2013) Quantitative SPECT/CT. Recent Results Cancer Res 187:313-330. doi:10.1007/978-3-642-10853-2_10

52. Bailey DL, Willowson KP (2013) An evidence-based review of quantitative SPECT imaging and potential clinical applications. J Nucl Med 54:83-89. doi:10.2967/jnumed.112.111476

53. Beauregard JM, Hofman MS, Pereira JM, Eu P, Hicks RJ (2011) Quantitative $\left({ }^{177}\right) \mathrm{Lu}$ SPECT (QSPECT) imaging using a commercially available SPECT/CT system. Cancer Imaging 11:56-66. doi:10.1102/1470-7330.2011.0012

54. Sandström M, Garske-Román U, Granberg D, Johansson S, Widström C, Eriksson B, Sundin A, Lundqvist H, Lubberink M (2013) Individualized dosimetry of kidney and bone marrow in patients undergoing ${ }^{177} \mathrm{Lu}$-DOTA-octreotate treatment. J Nucl Med 54:33-41. doi:10.2967/jnumed.112.107524

55. Lu SJ, Gnanasegaran G, Buscombe J, Navalkissoor S (2013) Single photon emission computed tomography/computed tomography in the evaluation of neuroendocrine tumours: a review of the literature. Nucl Med Commun 34:98-107. doi:10. 1097/MNM.0b013e32835bd59d

56. Schillaci $O$ (2007) Somatostatin receptor imaging in patients with neuroendocrine tumors: not only SPECT? J Nucl Med 48:498-500

57. Bombardieri E, Coliva A, Maccauro M, Seregni E, Orunesu E, Chiti A, Lucignani G (2010) Imaging of neuroendocrine tumours with gamma-emitting radiopharmaceuticals. Q J Nucl Med Mol Imaging 54:3-15 